\title{
MICROWAVE INSTABILITY AND IMPEDANCE MODEL
}

\author{
A. Mosnier, SOLEIL, Gif/Yvette (France)
}

\section{Abstract}

Tracking simulations, with the aim of studying the microwave regime with short and intense bunches, suggest different instability mechanisms, according to the impedance model. In order to get a better insight of the source of the instability, i.e. azimuthal or radial mode coupling, we chose to follow the Vlasov-Sacherer approach to investigate the stability of the stationnary solution. The generalized Sacherer's integral, including mode coupling and potential well distortion, was then solved by using the "step function technique" for the expansion of the radial function, as proposed by Oide and Yokoya. For illustration, the effect of the resonant frequency of a broadband resonator in the SOLEIL storage ring was studied. When the resonator frequency is much higher than the bunch spectrum width, azimuthal mode coupling can occur before radial mode coupling. When the resonator frequency is lower, radial mode coupling comes usually first, but two or more bunchlets are produced at relatively low current. The diffusion process between the bunchlets, which leads to the well-known "saw-tooth" behaviour, originates actually from a fast growing microwave instability. Lastly, the beneficial effect of an harmonic cavity on the microwave instability is estimated and discussed.

\section{INTRODUCTION}

The Vlasov-Sacherer approach is chosen to investigate the onset of the microwave instability, leading to abnormal bunch-lengthening and energy spread widening in electron storage rings. As soon as the potential well distorsion, due to wakefields induced in the vacuum chamber, is significant, single-particle trajectories are no longer ellipses and the spread in synchrotron frequency must be taken into account in the stability study of the stationnary distribution, given by the Haissinski's equation [1]. The Sacherer's integral equation with mode coupling [2] is then generalized to [3]

$$
\begin{aligned}
& (\Omega-m \omega(J)) R_{m}(J)= \\
& \quad-m \omega(J) \psi_{o}(J) k \sum_{m^{\prime}} \int G_{m m^{\prime}}\left(J, J^{\prime}\right) R_{m^{\prime}}\left(J^{\prime}\right) d J^{\prime}
\end{aligned}
$$

As the synchrotron motion is strongly nonlinear, the action-angle variables $(J, \phi)$ have been used. $\psi_{0}$ and $\omega$ are the amplitude-dependent distribution and synchrotron frequency of the equilibrium state. The perturbation distribution oscillates with the coherent frequency $\Omega$ and has been expanded into the usual radial functions $R_{m}$. The Kernel is an integral over the frequency, involving the impedance :

$$
G_{m m^{\prime}}\left(J, J^{\prime}\right)=\int \frac{Z(\omega)}{i \omega} K_{m}^{*}(\omega, J) K_{m^{\prime}}\left(\omega, J^{\prime}\right) d \omega
$$

where $K_{m}$ is an integral over the phase variable. The integral equation (1) was solved by expanding the radial function $R_{m}$.

$$
R_{m}(J)=\sum_{n} C_{m n} h_{n}(J)
$$

according to the "mesh technique", as suggested by Oide and Yokoya [4]. This method uses step functions for $h_{n}(J)$ which takes the constant value $1 / \Delta J_{n}$ in the strip around the $n$-th mesh with the thickness $\Delta J_{n}$, and zero elsewhere, and converts (1) into an eigenvalue's problem..

For illustration, the parameters of the SOLEIL storage ring [5] are used throughout the paper, as well as a broadband resonator as impedance model of the vacuum chamber. The shunt impedance was fixed to $3.6 \mathrm{k} \Omega$, but since the feature of the impedance, as seen by the beam, changes with the resonant frequency, we resolved to vary the resonator frequency on a wide frequency range, from 10 to $30 \mathrm{GHz}$, in order to study the effect on the phase space topology and, above threshold, on the origin of the microwave instability.

\section{HIGH RESONANT FREQUENCY}

The potential well distortion is first calculated by solving the Haissinski's equation for a $30 \mathrm{GHz}$ resonator (Fig.1).
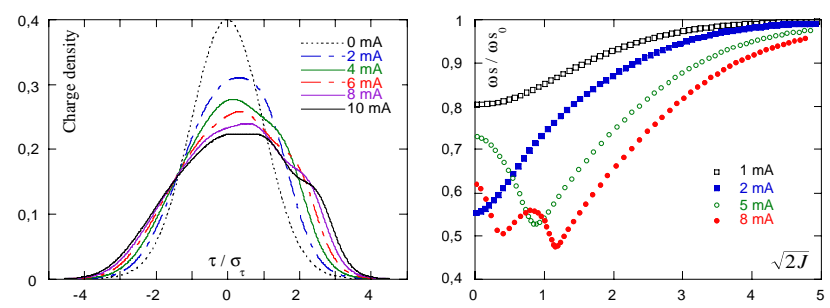

Figure 1: Charge distributions (top) - bunch head on the right side - and synchrotron frequency (bottom) for different beam currents (30 GHz resonator).

As usual, the distribution becomes asymmetric due to the resistive part of the impedance and the bunch shifts forward (positive momentum compaction) to compensate for the energy loss. The actual synchrotron frequency starts below the zero-current frequency due to the inductive part of the impedance. However, above $3 \mathrm{~mA}$, instead of raising monotically towards unity as the amplitude increases, it reaches a minimum due to the resistive component.

The next task consists in studying the stability of the stationary distribution. Looking first for eventual radial mode coupling by calculating the eigenvalues for each azimuthal mode $m$, separately, we find that the modes 
$m=3$ to 5 are unstable above $5 \mathrm{~mA}$. Fig. 2 shows for example the power spectra of the sextupole mode, calculated by means of the eigenvectors just before and after the instability threshold. The overlap of the resistance with the power density is higher at positive frequencies than at negative frequencies above $5.5 \mathrm{~mA}$, confirming the emergence of a radial mode coupling. If now we pursue the analysis by solving the system with pairs of azimuthal modes, we find a strong coupling between the modes $m=1$ and $m=2$ at relatively low intensity

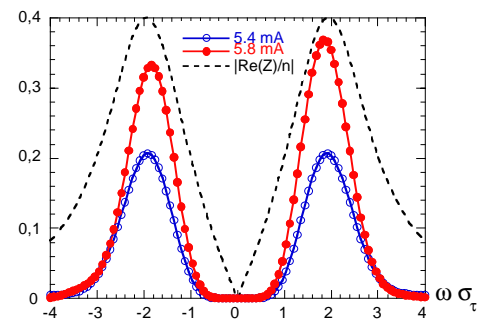

Figure 2: Power spectra the $m=3$ mode just before and after the instability threshold of $5.5 \mathrm{~mA}$.

Lastly, the coherent frequency is plotted in Fig.3, when more azimuthal modes than enough are taken into account ( $m=1$ to 6 ). A complete mixing occurs at relatively low current, after a rapid spread. The growth rate increases dramatically with a current around $5 \mathrm{~mA}$, which represents the onset of the instability. For higher intensity, the growth rate is larger than the radiation damping rate of the SOLEIL ring. Several types of instability - identified by solid circles - develop simultaneously, the nature of the most unstable modes changing with intensity : at the threshold of $5 \mathrm{~mA}$, the microwave instability is induced by a radial coupling of the sextupole mode and a coupling of the dipole and quadrupole modes; these instabilities are finally overtaken by the radial $m=5$ mode coupling above $8 \mathrm{~mA}$; an octupole mode can be also identified, but with a smaller growth rate.
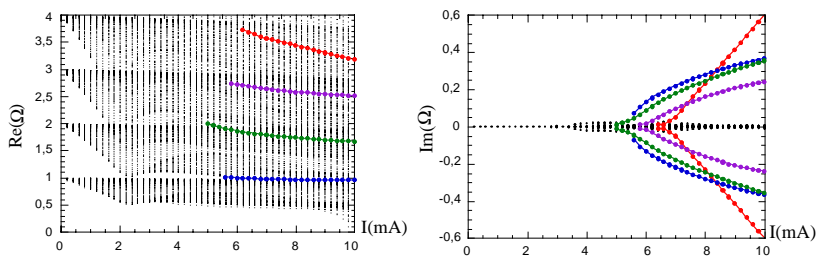

Figure 3: $\operatorname{Re}(\Omega)$ and $\operatorname{Im}(\Omega)$ vs. current ( $m=1$ to 6$)$.

\section{LOW RESONANT FREQUENCY}

Similarly, potential well distortion is first calculated for a lower resonant frequency $11 \mathrm{GHz}$ resonator (Fig.4). The bunch becomes much more distorted than before and two peaks appear above $3.5 \mathrm{~mA}$, as soon as there are two or more stable fixed points, forming distinct islands in the phase space (Fig.5). We note that the synchroton frequency is vanishing on the separatrix, whereas it is about equal to the zero-current frequency at the center of the first island and twice the zero-current frequency at the center of the second island.
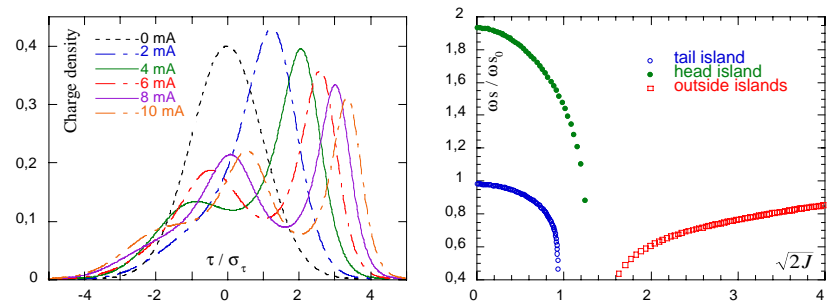

Figure 4: Charge distributions (top) - bunch head on the right side - and synchrotron frequency (bottom) for different beam currents (11 GHz resonator).
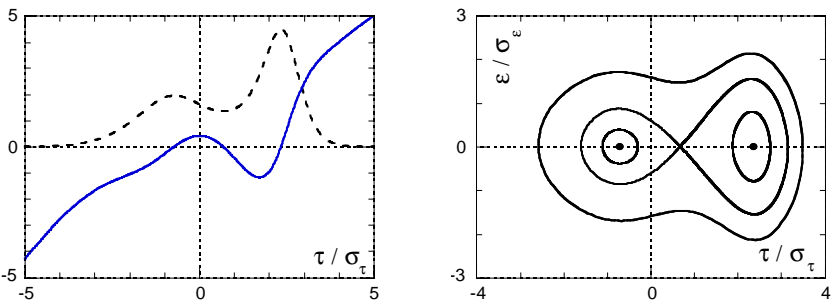

Figure 5: Norm. net voltage (left - solid line) and constant- $H$ contours in phase space (right) at $5 \mathrm{~mA}$.

The next task consists in studying the stability of the stationary distribution. The imaginary and real parts of $\Omega$, calculated for with a sufficient number of modes, are plotted as a function of bunch current in Fig.6. The growth rate looks more chaotic than for the higher frequency resonator, because of the rapid change of the topology of the phase space, perturbated by the formation of two or more bunchlets. Above $4 \mathrm{~mA}$, which can be considered as a threshold, two mode families with regular growth rate increase (identified by solid circles on the figure), stand out nevertheless. It is worth noting the sudden change of behaviour at a current of $6 \mathrm{~mA}$.
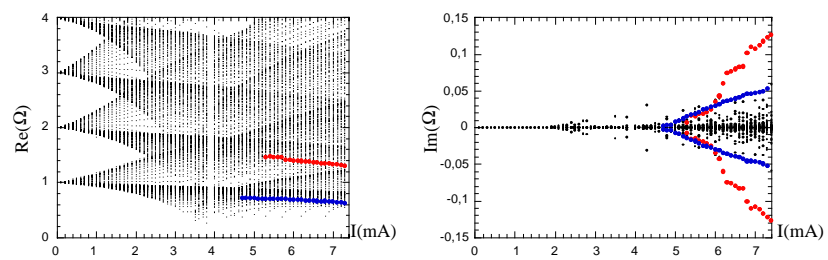

Figure 6: $\operatorname{Re}(\Omega)$ and $\operatorname{Im}(\Omega)$ vs. current ( $m=1$ to 6$)$.

Again, tracking simulations confirmed a threshold of 4 $\mathrm{mA}$, although some premonitory fluctuation of energy spread can be observed slightly before, as predicted. However, the so-called sawtooth instabilty, already observed in existing machines appears suddenly at $6 \mathrm{~mA}$. Tracking results show a quick increase of both energy spread and bunch length, followed by a slower decrease, with a recurrence of about $150 \mathrm{~Hz}$ (Fig. 7). 


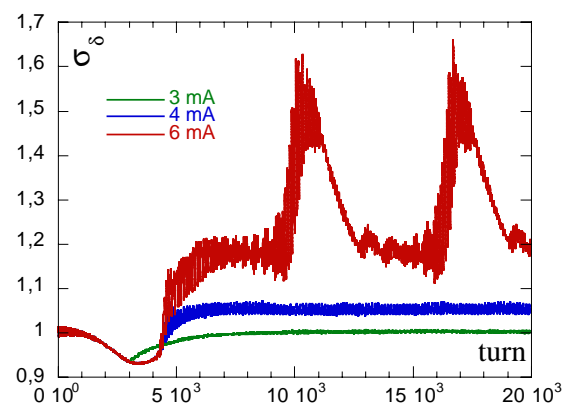

Figure 7: Energy spread widening as a function of the number of turns.

A density-plot of the most unstable distribution, calculated from the eigenvectors, is given in Fig.8 at the limit of emergence of the sawtooth behaviour $(6 \mathrm{~mA})$. The azimuthal pattern reveals a pure dipole mode inside the trailing bunchlet. It is worthwhile noting that this unstable dipole mode widens so far as to reach the separatrix of the tail island. Particles can diffuse through the unstable fixed point and populate the head bunchlet, leading to relaxation oscillations. A phenomenological description of the sawtooth behaviour was suggested in [6], but the diffusion process was assumed to originate from the random emission of radiation, instead of a strong instability.

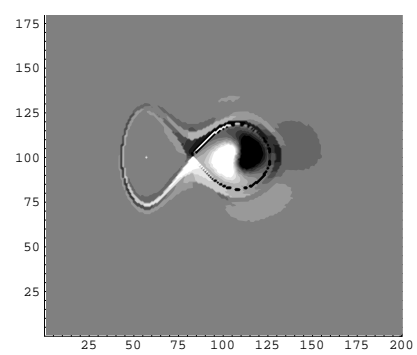

Figure 8: Density-plot of the dipole mode (6 mA).

\section{CONCLUSION}

The threshold of the microwave instability has been estimated over a wide frequency range of the broadband resonator. Although the source of the instability, radial or azimuthal mode coupling, is changing and although the azimuthal mode number is differing greatly (from $m=1$ or 2 at low frequency to $m=5$ or 6 at high frequency), the onset of the instability does not change a lot from $5 \mathrm{GHz}$ to $30 \mathrm{GHz}$. It is plotted in Fig. 9 as a function of the frequency $\omega_{r} \sigma_{\tau}$, and has a broad minimum between 1 and 1.5. However, the threshold is not the only criterion; in particular, lower frequency resonators are more harmful since they can induce dipole or quadrupole oscillations of large amplitude and sawtooth type instabilities can develop, owing to the formation of micro-bunches.

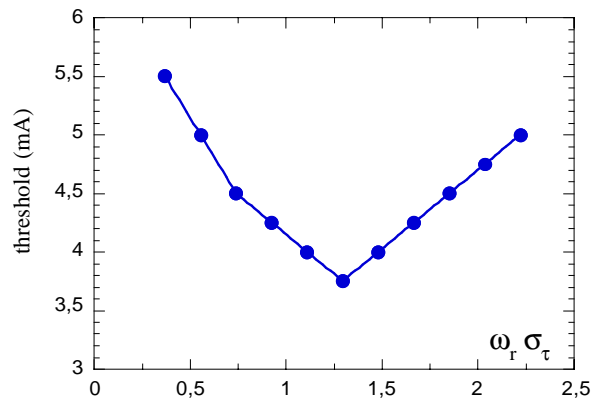

Figure 9: Microwave instability threshold as function of normalized resonator frequency.

Although the primary goal of an harmonic cavity, operating in the bunchlengthening mode, is to increase beam lifetime in Synchrotron Light Sources, it has also a beneficial effect on the microwave instability. As the use of the harmonic cavity reduces strongly the peak current, we could expect a large increase of the instability threshold. Besides, since the final voltage, including the wake potential, is smoothed off, it will suppress multiple bunchlets, which would appear at relatively low current. However, we found [3] that, even though the particle density is divided by a factor of about 4 , the instability threshold enhancement is only a factor two. This efficiency loss can be explained by the lower synchrotron frequency spread due to a lower potential well distortion (Fig.10). In case of short bunches, the non-linearity and then the Landau damping effect of an harmonic cavity, even operating at the third harmonic, is much smaller than the wakefield's one.
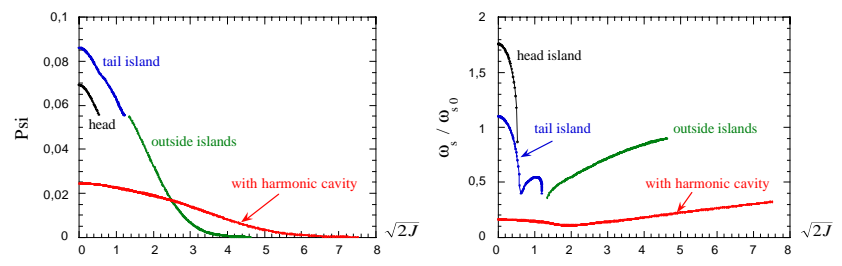

Figure 10:Magnitude $\psi_{0}$ (left) and synchrotron frequency (right) of the stationnary distribution vs. $\sqrt{ } 2 \mathrm{~J}$.

\section{REFERENCES}

[1] J. Haissinski, II Nuovo Cimento 18 B, 72 (1973).

[2] F. Sacherer, "Bunch lengthening and microwave instability", IEEE Trans. Nucl. Sci., NS-24, 1393 (1977).

[3] A. Mosnier, "Microwave Instability Study in the SOLEIL Ring", SOLEIL Report 98-11, 1998.

[4] K. Oide and K. Yokoya, "Longitudinal single-bunch instability in electron storage rings", KEK Preprint 90-10, April 1990.

[5] M.P. Level et al, "Status of the SOLEIL Project", Proc. of the 6th European Part. Acc. Conf., Stockholm, June 1998.

[6] R. Baartman and M. D'Yachkov, "Simulations of Sawtooth Instability", PAC95, 3119 (1995). 